\title{
Ten years risk assessment of Atherosclerotic \\ cardiovascular disease using Astro-CHARM and Pooled Cohort Equation in a South Asian population
}

Tariq Ashraf ( $\square$ tariqash45@gmail.com )

National Institute of Cardiovascular Diseases, Karachi, Pakistan

Muhammad Naeem Mengal

National Institute of Cardiovascular Diseases, Karachi, Pakistan

Atif Sher Muhammad

National Institute of Cardiovascular Diseases, Karachi, Pakistana

Asal Khan Tareen

National Institute of Cardiovascular Diseases, Karachi, Pakistan

Muhammad Nauman Khan

National Institute of Cardiovascular Diseases, Karachi, Pakistan

Khawar Kazmi

National Institute of Cardiovascular Diseases, Karachi, Pakistan

Asif Nadeem

Combined Military Hospital (CMH), Malir, Karachi, Pakistan

Shakil Sarwar

Combined Miliray Hospital (CMH), Malir, Karachi, Pakistan

\section{Zara Bashir}

Aga Khan University Hospital, Karachi, Pakistan

\section{Nadeem Qamar}

National Institute of Cardiovascular Diseases, Karachi, Pakistan

Musa Karim

National Institute of Cardiovascular Diseases, Karachi, Pakistan

\section{Research article}

Keywords: Atherosclerotic Cardiovascular Disease, Astro-CHARM, Pooled Cohort Equation

Posted Date: December 11th, 2019

DOI: https://doi.org/10.21203/rs.2.18654/v1

License: @ (i) This work is licensed under a Creative Commons Attribution 4.0 International License. Read Full License 
Version of Record: A version of this preprint was published at BMC Public Health on March 27th, 2020. See the published version at https://doi.org/10.1186/s12889-020-08472-4. 


\section{Abstract}

Background Atherosclerotic cardiovascular diseases (ASCVD) are on the rise in low and middle income countries attributed to modern sedentary lifestyle and dietary habits. This has led to need of assessment of the burden of atrisk population so that prevention measures can be developed. The objective of this study was to assess ten years risk assessment of ASCVD using Astro-CHARM and Pooled Cohort Equation (PCE) in a South Asian population.

Methods A total of 386 residents of all six districts of Karachi with no ASCVD were enrolled in the study. The inclusion criteria consisted of age 40 years or above and either gender. Study participants were enrolled after obtaining informed written consent and those study participants who were found to have either congenital heart disease or valvular heart diseases or ischemic heart disease were excluded from the study based on initial screening. For the calculation of 10 years risk of ACVD based on Astro-CHARM and PCE, the variables were obtained including medical history and coronary artery calcium and C-reactive protein measurements.

Results Mean estimated 10-year risk of fatal or non-fatal myocardial infarction or stroke as per the Astro-CHARM was $13.98 \pm 8.01 \%$, while Mean estimated 10-year risk of fatal or non-fatal myocardial infarction or stroke as per the PCE was $22.26 \pm 14.01 \%$. Based on Astro-CHARM, $11.14 \%$ of the study participants were labelled as having high risk, while PCE estimated $20.73 \%$ of study participants as having high risk of ASCVD.Conclusion Despite the fact that our findings showed substantial differences in ten-year risk of ASCVD between Astro-CHARM and PCE, both calculators can be used to develop a new population specific risk estimator for the South Asian population. Our study provides the first step towards developing a risk assessment guided decision making protocol for primary prevention of ASCVD for South Asian population.

\section{Introduction}

With the ever increasing global burden of atherosclerotic cardiovascular diseases (ACVD) the need to timely assess the risk of a cardiovascular events cannot be more emphasized (1-5). Once among the most common causes of death in high income countries only, ACVD has become the leading cause of morbidity and mortality in low and middle income countries as well owing to epidemiologic transition and changing population dynamics. This trend is more prominent in South Asian population where (ACVD) is the leading cause of death $(6,7)$.

A number of risk estimators have been developed to assess the risk of any cardiovascular event in at risk population. Astro-CHARM and Pooled Cohort Equation (PCE) has been much widely used worldwide however these estimators have been developed in the west and not much evidence is present that how accurately they estimate ACVD in South Asian population.(8, 9). Tariq et al. (10) reported that Pakistani population showed to have higher risk of ASCVD compared to whites, blacks, and Hispanic and even South Asians living in USA. Another study conducted in similar population showed that the burden of premature myocardial infarction to be as high as $12 \%$ (11). In addition, another large multi-centric study from the Pakistan attempted to predict the risk of atherosclerotic cardiovascular disease in Pakistani population, however, included subject with already suffered from ASCVD (12). Such methodological flaws can have serious implications while projecting risk assessment on the general population. There is a scarcity of literature providing estimates of ASCVD risk in resident South Asian population as well as comparison of different risk estimators in this population.

Majority of these studies have used PCE as risk estimator which has its own limitation that includes overestimating the risk in other nationalities/ ethnicities and in population with less social deformation which 
requires attention while making medical decisions. To overcome this Astro-CHARM calculation was used (Astronaut Cardiovascular Health and Risk Modification). Data in white, Hispanic \& black have been elucidated except for the south Asians.(8)

This study, to the best of our knowledge attempted to estimate the ten year risk of ASCVD in general population without any history of ASCVD using both Astro-CHARM and PCE, and compared the estimated risks using both.

\section{Methods}

This analytical cross-sectional study was conducted among three hundred and eighty-six of all six districts of Karachi who had no prior history of atherosclerotic cardiovascular disease including stroke. The selection of the study participants were done through snowball sampling. The inclusion criteria consisted of age 40 years of above and either gender. Study participants were enrolled after obtaining informed written consent and those study participants who were found to have either congenital heart disease or valular heart diseases or ischemic heart disease were excluded from the study based on initial screening.

Continuous data was assessed for normality based on visual inspection in order to apply parametric tests for comparisons. For the calculation of 10 years risk of ACVD based on Astro-CHARM and PCE, the variables were obtained in the following manner. Diabetes mellitus, hypertension, current smoking, and family history of heart attack were defined as per Astro-CHARM definitions (8).

High-sensitivity C-reactive protein (hs-CRP) levels were measured by using the following assays. MESA, BNII nephelometer (N High Sensitivity CRP; Dade Behring Inc); DHS, Roche/Hitachi 912 System, Tina-quant assay (Roche Diagnostics), alatex-enhanced immunoturbidimetric method; PACC, particleenhancedimmune turbidimetric latex agglutination assay; FHS, enhanced immune turbidimetric high-sensitivity assay(Roche Diagnostics). CT angio was performed in all the patients and coronary artery calcium (CAC) score was calculated for all the patients.

Data were entered using MS Excel for Windows and analyses were done using STATA 11.0 while 10 years risk of ACVD was calculated using Astro-CHARM and PCE using the online calculators available at www.AstroCHARM.org and www.clincalc.com respectively. Participants were stratified as low-risk and high-risk individuals based on cutoff have of $\geq 7.5 \%$ for both Astro-CHARM and PCE.

Descriptive analyses were conducted by calculating the mean and standard deviation for all continuous variables while frequency and percentages were calculated for all categorical variables. Risk of ACVD was reported using both equations for combined study population and were also stratified for different sub-groups.

\section{Results}

Mean age of the study participants was $49 \pm 7.10$ years with $54 \%$ Males. Mean BMI was $28.11 \pm 5.38$ while $45.6 \%$, $15.8 \%$ and $14.2 \%$ were hypertensives, diabetics and current smokers respectively. Six (two percent) of the study participants had all three co-morbid conditions. Thirty percent had positive family history of MI. Mean CAC was

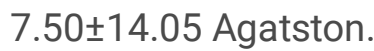

Mean estimated 10-year risk of fatal or non-fatal myocardial infarction or stroke as per the Astro-CHARM was $13.98 \pm 8.01 \%$, while Mean estimated 10-year risk of fatal or non-fatal myocardial infarction or stroke as per the 
PCE was 22.26 $\pm 14.01 \%$ shown in Figures 1 and 2. Risk comparison in different age categories showed significantly higher risk of ACVD in age groups of 55-60 years and 60 years and above compared to the ASCVD risk in the age group 40-45 years as calculated per Astro-CHARM. Table 1 shows the post-hoc comparisons of risk between different age groups as per Astro-CHARM. Figure 4 shows the comparison of percentages of individuals categorized as high risk using both estimators.

Similarly table $2 \mathrm{a}$ and $2 \mathrm{~b}$ below shows the comparison of baseline characteristics of high and low risk individuals based on Astro-CHARM and Pooled Cohort Estimator. Kappa agreement between the two calculators in terms of labelling high and low risk individuals was high $(0.50$, p-value $<.0001)$ and PCE correctly labelled $87.75 \%$ of the individuals.

\section{Discussion}

To the best of our knowledge, current study reports ten year risk of ASCVD among non-cardiac Pakistani population using both Astro-CHARM and PCE and the comparison of the two calculators for the first time. In concordance with the findings of other studies, PCE overestimated the risk of ASCVD in the study population almost twice as that estimated by Astro-CHARM. Astro-CHARM which also includes indicators like coronary artery calcium and C-reactive protein during ASCVD risk assessment, these indicators are not readily available in low and middle-income countries therefore the significance of PCE cannot be neglected (13).

One of the key findings of this study was that both coronary artery calcium and $\mathrm{C}$ reactive protein significantly correlated with the risk scores generated by both Astro-CHARM and PCE which is in concordance with findings from other studies $(14,15)$. Especially in the case of CAC, a relatively recent study by JJ Carr et al (14) reported its effect on the risk of ASCVD among individuals well below 40 years of age. Keeping in mind that said study was conducted among African-Americans and Caucasians, who are relatively at lower risk of developing ASCVD compare to South Asians, it points towards a major limitation of these risk calculators among South Asians, who, in clinical experience suffer cardiac events at an even younger age (16) and evidenced by previous study from Pakistan (10), there is a dire need of development of population specific risk estimator for the South Asian population. This study provided the grounds towards developing such risk estimator as well as initial step towards developing evidence-based decision making for primary prevention guidelines of atherosclerotic cardiovascular disease in this population.

Considering the fact that the population of South Asian countries consist of multi-ethnical groups with possibly different inherent risk of developing ASVD, as well as the absence of risk estimators in population below 40 years of age much evidence is required to develop population specific risk estimators and risk guided decision making tools for primary prevention among South Asians using robust study designs. Furthemore, considering the suboptimal healthcare systems and absence of expensive investigating facilities like CAC in low and middle income countries of South Asia, development of risk estimators for these population also require large population based, ethnicity and age specific data to develop such a tool using both Astro-CHARM and PCE. Low and middle income countries are now overburdened with interventional procedures like angioplasty, and expensive methods of secondary prevention. Accurate risk assessment is the only solution in such resource poor settings where primary prevention can be implicated through lifestyle modifications and statins among high risk individuals. 


\section{Conclusion}

Despite the fact that our findings showed substantial differences in ten-year risk of ASCVD between Astro-CHARM and PCE, both calculators can be used to develop a new population and specific risk estimators for the South Asian population. Our study provides the first step towards developing a risk assessment guided decision making protocol for primary prevention of ASCVD for South Asian population.

STRENGTHS AND LIMITATIONS:

The biggest strength of the study is the use and comparison of both ASCVD estimators in non-cardiac Pakistani general population selected from the largest city of the country with diverse population sub-groups from entire country thus providing data facsimile to general population of Pakistan.

Major limitation of the study is lies in the study design where lack of prospective follow-up cannot ensure the accuracy of these estimates. Furthermore, the data was taken from urban setting and implications of the findings cannot be ascertained on rural population.

\section{Declaration}

\section{Ethics approval and consent to participate}

The ethical approval for the study was obtained from the Ethical Review Committee of National Institute of CardioVascular Diseases (NICVD), Karachi, Pakistan.

\section{Consent for publication}

The manuscript does not contain any individual's identifiable information or data

\section{Competing interests}

The authors declare that there are no competing interests.

\section{Funding}

Computed Tomography (CT) angiography and laboratory assessments were financed by Medical Affairs division of Getz Pharma.

\section{Authors' contributions}

TA conceptualized the study and supervised the entire project, MNM, ASM, AKT, MNK and KAK conducted the study and data collection from patients, AN and SS conducted the data collection and facilitated CT angiography, ZB provided technical support while NQ and MK conducted the statistical analyses. All authors were involved and contributed at each step of manuscript writing.

\section{Data availability}

The data for the current study are available on request

\section{Acknowledgements}


Authors are thankful to the Medical Affairs division of Getz Pharma for providing financial support for CT angiography and lab assessments of the study participants. The authors wish to acknowledge the support of the staff member of the clinical research department of the National Institute of Cardiovascular Diseases (NICVD), Karachi Pakistan.

\section{References}

1. Barquera S, Pedroza-Tobías A, Medina C, Hernández-Barrera L, Bibbins-Domingo K, Lozano R, et al. Global overview of the epidemiology of atherosclerotic cardiovascular disease. Archives of medical research. 2015;46(5):328-38.

2. Cappuccio FP, Miller MA. Cardiovascular disease and hypertension in sub-Saharan Africa: burden, risk and interventions. Internal and emergency medicine. 2016;11(3):299-305.

3. Lackland DT, Weber MA. Global burden of cardiovascular disease and stroke: hypertension at the core. Canadian Journal of Cardiology. 2015;31(5):569-71.

4. Roth GA, Johnson C, Abajobir A, Abd-Allah F, Abera SF, Abyu G, et al. Global, regional, and national burden of cardiovascular diseases for 10 causes, 1990 to 2015. Journal of the American College of Cardiology. 2017;70(1):1-25.

5. Roth GA, Johnson CO, Abate KH, Abd-Allah F, Ahmed M, Alam K, et al. The burden of cardiovascular diseases among US states, 1990-2016. JAMA cardiology. 2018;3(5):375-89.

6. Volgman AS, Palaniappan LS, Aggarwal NT, Gupta M, Khandelwal A, Krishnan AV, et al. Atherosclerotic cardiovascular disease in South Asians in the United States: epidemiology, risk factors, and treatments: a scientific statement from the American Heart Association. Circulation. 2018;138(1):e1-e34.

7. Goyal A, Yusuf S. The burden of cardiovascular disease in the Indian subcontinent. Indian J Med Res. 2006;124(3):235-44.

8. Khera A, Budoff MJ, O’Donnell CJ, Ayers CA, Locke J, de Lemos JA, et al. Astronaut Cardiovascular Health and Risk Modification (Astro-CHARM) coronary calcium atherosclerotic cardiovascular disease risk calculator. Circulation. 2018;138(17):1819-27.

9. Karmali KN, Goff DC, Ning H, Lloyd-Jones DM. A systematic examination of the 2013 ACC/AHA pooled cohort risk assessment tool for atherosclerotic cardiovascular disease. Journal of the American College of Cardiology. 2014;64(10):959-68.

10. Ashraf T, Achakzai AS, Farooq F, Memon MA, Mengal N, Abbas KY, et al. Estimating risk of atherosclerotic cardiovascular diseases in non-atherosclerotic Pakistani patients: study conducted at National Institute of Cardiovascular Diseases, Karachi, Pakistan. JPMA J Pak Med Assoc. 2017;67(April (4)):494-8.

11. Batra MK, Rizvi NH, Sial JA, Saghir T, Karim M. Angiographic characteristics and in hospital outcome of young patients, age up to 40 versus more than 40 years undergoing primary percutaneous coronary intervention. JPMA. 2019.

12. Hassan K, Mohydin B, Fawwad A, Waris N, Iqbal S, Jawaid M. Predicting the risk of atherosclerotic cardiovascular disease (ASCVD) in Pakistani population. Clinical Epidemiology and Global Health. 2019;7(2):184-7.

13. Blaha MJ, Cainzos-Achirica M, Greenland P, McEvoy JW, Blankstein R, Budoff MJ, et al. Role of coronary artery calcium score of zero and other negative risk markers for cardiovascular disease: the Multi-Ethnic Study of Atherosclerosis (MESA). Circulation. 2016;133(9):849-58. 
14. Carr JJ, Jacobs DR, Terry JG, Shay CM, Sidney S, Liu K, et al. Association of coronary artery calcium in adults aged 32 to 46 years with incident coronary heart disease and death. JAMA cardiology. 2017;2(4):391-9.

15. Cozlea D, Farcas D, Nagy A, Keresztesi A, Tifrea R, Cozlea L, et al. The impact of $C$ reactive protein on global cardiovascular risk on patients with coronary artery disease. Current health sciences journal. 2013;39(4):225.

16. Bilen O, Kamal A, Virani SS. Lipoprotein abnormalities in South Asians and its association with cardiovascular disease: Current state and future directions. World journal of cardiology. 2016;8(3):247.

\section{Tables}

Table 1 Baseline socio-demographic and medical characteristics of study participants $n=386$

\begin{tabular}{lr}
\hline \multicolumn{3}{c}{ Baseline characteristics } \\
\hline Weight $(\mathrm{kg})^{*}$ & $73.53 \pm 14.45$ \\
\hline Height $(\mathrm{cm})^{*}$ & $162.01 \pm 10.83$ \\
\hline Age (years)* & $49.00 \pm 7.11$ \\
\hline Coronary artery calcium (Agatston units)* & $31.57 \pm 195.63$ \\
\hline Total cholesterol (mg/dL)* & $177.65 \pm 38.97$ \\
\hline HDL cholesterol (mg/dL)* & $51.73 \pm 36.77$ \\
\hline Systolic blood pressure (mmHg)* & $138.83 \pm 21.23$ \\
\hline Gender & \\
Male & $211(54.7 \%)$ \\
Female & $175(45.3 \%)$ \\
\hline Hypertensive & $176(45.6 \%)$ \\
Yes & $210(54.4 \%)$ \\
No & \\
\hline Diabetic & $61(15.8 \%)$ \\
Yes & $325(84.2 \%)$ \\
No & \\
\hline Currentsmoker & $55(14.2 \%)$ \\
Yes & $118(30.6 \%)$ \\
No & $268(69.4 \%)$ \\
\hline Family History of Myocardial Infarction & \\
Yes & \\
No & \\
\hline
\end{tabular}

* reported as mean and standard deviation, all othervariables are reported as frequencies and percentages

Table 2aComparison of baseline characteristics of high and low risk individualsbased on AstroCHARM and Pooled Cohort Estimator for all continuous variables 


\begin{tabular}{|c|c|c|c|c|c|c|c|c|}
\hline & \multicolumn{4}{|c|}{ Astro-CHARM } & \multicolumn{4}{|c|}{ Pooled Cohort Estimator } \\
\hline & \multicolumn{2}{|c|}{ Low Risk } & \multicolumn{2}{|c|}{ High Risk } & \multicolumn{2}{|c|}{ Low Risk } & \multicolumn{2}{|c|}{ High Risk } \\
\hline & Mean & Standard & Mean & Standard & Mean & Standard & Mean & Standard \\
\hline & & Deviation & & Deviation & & Deviation & & Deviation \\
\hline Weight (kg) & 73 & 15 & 74 & 14 & 74 & 15 & 72 & 13 \\
\hline Height $(\mathrm{cm})$ & 162 & 11 & 165 & 10 & 161 & 11 & 166 & 10 \\
\hline Age (years) & 48 & 7 & 55 & 7 & 47 & 6 & 57 & 6 \\
\hline CoronaryArteryCalcium(Agatston) & 9.93 & 46.42 & 204.17 & 546.76 & 15.52 & 73.87 & 92.96 & 400.79 \\
\hline Total Cholesterol (mg/dL) & 178 & 37 & 178 & 50 & 175 & 39 & 187 & 37 \\
\hline HDL Cholesterol (mg/dL) & 53 & 39 & 45 & 10 & 53 & 41 & 45 & 11 \\
\hline Systolic Blood Pressure (mmHg) & 137 & 20 & 155 & 21 & 137 & 20 & 148 & 22 \\
\hline
\end{tabular}

\section{Table 2b}

\begin{tabular}{|c|c|c|c|c|c|c|c|c|c|}
\hline & & \multicolumn{4}{|c|}{ Astro-CHARM } & \multicolumn{4}{|c|}{ Pooled Cohort Estimator } \\
\hline & & \multicolumn{2}{|c|}{ Low Risk } & \multicolumn{2}{|c|}{ High Risk } & \multicolumn{2}{|c|}{ Low Risk } & \multicolumn{2}{|c|}{ High Risk } \\
\hline & & $\mathrm{n}$ & $\%$ & $\mathrm{n}$ & $\%$ & $\mathrm{n}$ & $\%$ & $\mathrm{n}$ & $\%$ \\
\hline \multirow[t]{2}{*}{ Gender } & Male & 178 & $51.9 \%$ & 33 & $76.7 \%$ & 145 & $47.4 \%$ & 66 & $82.5 \%$ \\
\hline & Female & 165 & $48.1 \%$ & 10 & $23.3 \%$ & 161 & $52.6 \%$ & 14 & $17.5 \%$ \\
\hline \multirow[t]{2}{*}{ Hypertensive } & Yes & 153 & $44.6 \%$ & 23 & $53.5 \%$ & 136 & $44.4 \%$ & 40 & $50.0 \%$ \\
\hline & No & 190 & $55.4 \%$ & 20 & $46.5 \%$ & 170 & $55.6 \%$ & 40 & $50.0 \%$ \\
\hline \multirow[t]{2}{*}{ Diabetic } & Yes & 38 & $11.1 \%$ & 23 & $53.5 \%$ & 27 & $8.8 \%$ & 34 & $42.5 \%$ \\
\hline & No & 305 & $88.9 \%$ & 20 & $46.5 \%$ & 279 & $91.2 \%$ & 46 & $57.5 \%$ \\
\hline \multirow[t]{2}{*}{ CurrentSmoker } & Yes & 38 & $11.1 \%$ & 17 & $39.5 \%$ & 28 & $9.2 \%$ & 27 & $33.8 \%$ \\
\hline & No & 305 & $88.9 \%$ & 26 & $60.5 \%$ & 278 & $90.8 \%$ & 53 & $66.2 \%$ \\
\hline \multirow[t]{2}{*}{ Family History of Myocardial infarction } & Yes & 98 & $28.6 \%$ & 20 & $46.5 \%$ & 98 & $32.0 \%$ & 20 & $25.0 \%$ \\
\hline & No & 245 & $71.4 \%$ & 23 & $53.5 \%$ & 208 & $68.0 \%$ & 60 & $75.0 \%$ \\
\hline
\end{tabular}

